www.nature.com/pj

\title{
EDITORIAL
}

\section{PJ ZEON Award for outstanding papers in Polymer Journal 2014}

Polymer Journal (2015) 47, 413-414; doi:10.1038/pj.2015.24

$T^{\mathrm{h}}$

The PJ ZEON Award started since 2005 as the successor of the J Paper Award.

The winners of the 2014 PJ ZEON Award have been announced by the Society of Polymer Science Japan (SPSJ) as follows.

Tomoki Ogoshi (Kanazawa University, Japan) for the contribution 'Extension of polyethylene chains by formation of polypseudorotaxane structures with perpentylated pillar[5]arenes', Vol. 46, No.1, 2014.

Xiaomei Shi (760th Research Institute China Shipbuilding Industry Corporation, China) for the contribution 'Gelation/crystallization mechanisms of UHMWPE solutions and structures of ultradrawn gel films', Vol. 46, No. 1, 2014.

Syuhei Yamaguchi (Hitachi Zosen Corporation, Japan) for the contribution 'Green polymer chemistry: lipase-catalyzed synthesis of biobased reactive polyesters employing itaconic anhydride as a renewable monomer', Vol. 46, No. 1, 2014.

Academic profiles of the winners can be found below this announcement.

Ogoshi, Shi and Yamaguchi received their award certificates and medals at an award ceremony held in conjunction with the SPSJ annual meeting in May 2015 in Hokkaido. Each winner also received a cash prize of 300000 JP yen and presented an invited talk based on their respective papers.

On behalf of the editors and editorial board of Polymer Journal, I wish to congratulate Ogoshi, Shi and Yamaguchi on this honor in recognition of their excellent papers. ${ }^{1-3}$ I hope the award will provide encouragement to these young researchers for their bright future career.

This PJ ZEON Award is open to all of the first authors of papers published in Polymer Journal. ${ }^{4}$ I am looking forward to receiving many applications for the 2015 PJ ZEON Award. Each year the SPSJ selects up to three most outstanding papers published by young authors in Polymer Journal, as recommended by the selection committee and board of directors of the SPSJ. Those who are interested should go to the SPSJ website (http://main.spsj. or.jp/) for further information. Finally, we express our sincere appreciation to Zeon Corporation for their generous sponsorship of this award.

Takashi Kato Editor-in-Chief E-mail: hpj@spsj.or.jp

\section{ABOUT THE WINNERS}

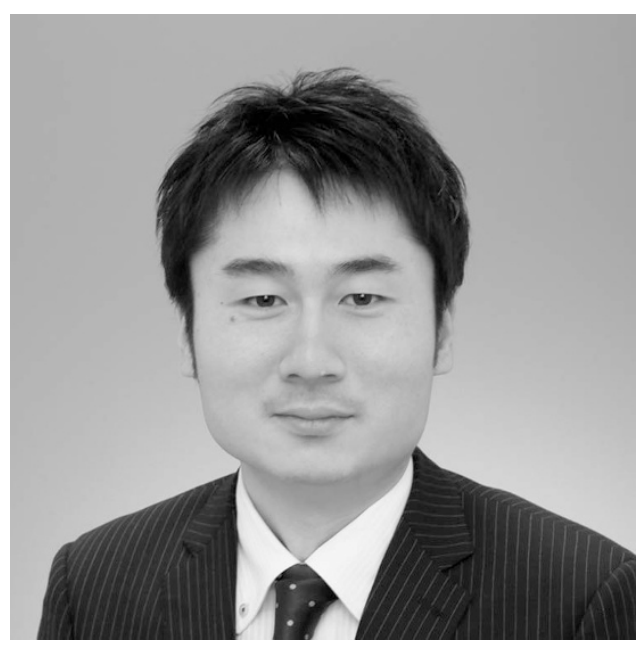

Tomoki Ogoshi received his PhD degree (2005) from Kyoto University under the supervision of Professor Yoshiki Chujo. He was a JSPS postdoctoral research fellow (2005-2006) in the Graduate School of Science at Osaka University in the group of Professor Akira Harada. In 2006, he moved to the Graduate School of Natural Science and Technology at Kanazawa University as an assistant professor and was promoted to an associate professor in 2010. His research interests include organic, polymer synthesis and supramolecular materials.

About the award article: The authors reported extension of highdensity polyethylene (HDPE) chains by formation of polypseudorotaxane structures with perpentylated pillar[5] arenes. Melt mixing of polymeric chains of HDPEs and wheels of perpentylated pillar[5] arenes resulted in formation of polypseudorotaxane structures. The formation of the polypseudorotaxane structures led to extension of the HDPE chains, which dramatically increased the melting point of the HDPE from 126 to $152^{\circ} \mathrm{C}$. The authors also demonstrated molten-tosolid and solid-to-molten state transitions of HDPE based on the host-guest system. HDPE was melted at $140^{\circ} \mathrm{C}$, but changed to a solid upon addition of perpentylated pillar[5] arenes. Further addition of competitive guest 1,4-dibromobutane to the solid mixture induced a solid-to-molten state transition. 


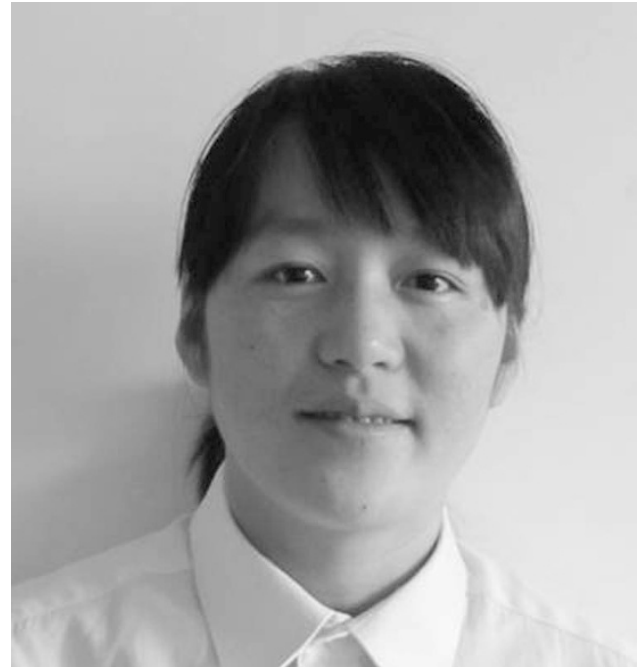

Xiaomei Shi has worked as one of the research members of 760th Research Institute China Shipbuilding Industry Corporation at Dalian of China since September 2013. She received BS at Qiqihaer University of China, MS at Xinjiang University and $\mathrm{PhD}$ at Dalian University of Technology. Her subjects mainly focus on (1) Biological functional gradient materials (preparation and characterization of artificial acetabulum) by using biopolymer and ultra-high molecular weight polyethylene (UHMWPE) composites; (2) research of hydrogel with high absorbent resins (organic-inorganic composite hydrogel, drug release); (3) layer-by-layer film preparation; (4) properties of surfactant in solution; and (5) gelation mechanism of UHMWPE solutions.

About the award article: The author reported that the galation mechanism of UHMWPE chains differed significantly in decalin and paraffin, although these are typically solvents used to gel spin UHMWPE fibers with high strength and moduli. In both solvents, gelation was attributed to liquid-liquid phase separation driven by concentration fluctuations during the initial stage. The gelation speeds and temperatures differed significantly in the two solvents. In decalin, solvent flowed from the gels, syneresis occurred crystallization and the dry-gel films were prepared. In contrast, in paraffin, many nucleating points appeared immediately and no syneresis occurred even after quenching to room temperature. To prepare dry-gel films, paraffin was blotted with filter paper and paraffin residues were removed in hexane. However, the small-angle X-ray scattering spectra from both films were similar, indicating crystalline lamellae and ultradrawing.

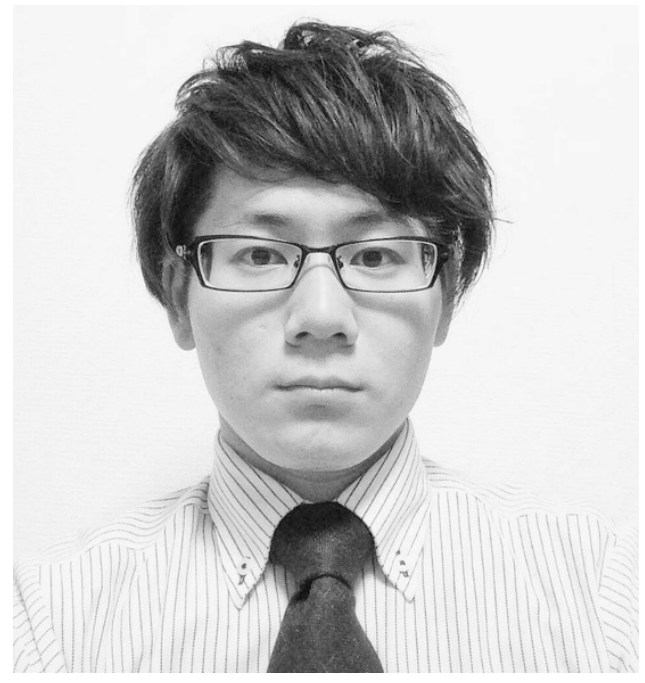

Syuhei Yamaguchi received his Master degree in 2013 from Kyoto Institute of Technology, and works in Hitachi Zosen Corporation. His research projects especially focus on enzyme-catalyzed synthesis of bio-based polymers and reaction mechanism.

About the award article: The authors reported lipase-catalyzed synthesis of bio-based reactive polyesters employing itaconic anhydride as a renewable monomer. Lipase (Novozym 435)-catalyzed ringopening addition condensation polymerization of reactive polyestersemployed itaconic anhydride (IAn), succinic anhydride (SAn), or glutaric anhydride (GAn) as starting monomer with combination of several diols. The product polyesters have a polymerizable vinylidene group derived from IAn. Model reaction gave useful information for regioselectivity of IAn and substrate selectivity of IAn toward SAn or GAn. Moreover, crosslinking reaction was attempted by using product polyester. IAn is a new substrate monomer for the lipase-catalyzed polymerization. The result of this study is the basis of enzymecatalyzed synthesis of bio-based polymers and possible of application as a new bio-based polymers.

1 Ogoshi, T., Kayama, H., Aoki, T., Yamagishi, T., Ohashi, R. \& Mizuno, M. Extension of polyethylene chains by formation of polypseudorotaxane structures with perpentylated pillar[5]arenes. Polym. J. 46, 77-81 (2014).

2 Shi, X., Bin, Y., Hou, D., Men, Y. \& Matsuo, M. Gelation/crystallization mechanisms of UHMWPE solutions and structures of ultradrawn gel films. Polym. J. 46, 21-35 (2014).

3 Yamaguchi, S., Tanha, M., Hult, A., Okuda, T., Ohara, H. \& Kobayashi, S. Green polymer chemistry: lipase-catalyzed synthesis of bio-based reactive polyesters employing itaconic anhydride as a renewable monomer. Polym. J. 46, 2-13 (2014).

4 Kato, T. PJ ZEON Award for outstanding papers in Polymer Journal 2013. Polym. J. 46 313-314 (2014) 